\title{
Adsorção de Cu e Zn num Latossolo Vermelho tratado com dejetos suínos ${ }^{1}$
}

\author{
Claudia Lopes ${ }^{2}$, Mari Lucia Campos ${ }^{3}$, Cristian Berto da Silveira ${ }^{4}$, Luciano Colpo Gatiboni, \\ David José Miquelutti ${ }^{6}$, Paulo Cezar Cassol ${ }^{7}$, Íris de Freitas Medeiros ${ }^{8}$ \\ http://dx.doi.org/10.1590/0034-737X201461060016
}

\section{RESUMO}

O Cu e $\mathrm{Zn}$ oriundos dos dejetos suínos podem se acumular no solo, oferecendo riscos para a saúde humana e a animal. O objetivo deste trabalho foi determinar a capacidade de adsorção e a competição de $\mathrm{Cu}$ e Zn num Latossolo Vermelho distroférrico, que recebeu aplicações continuadas de dejetos suínos, com taxa de aplicação de $200 \mathrm{~m}^{3}$ ha-1 ano $^{-1}$, durante sete anos, e comparar a adsorção com solo que nunca recebeu dejeto. Para avaliar a adsorção do Cu e $\mathrm{Zn}$, foram aplicados ao solo soluções de $\mathrm{Cu}$ e Zn, nas doses de $0,25,50,100,150,200,400,1.200 \mu \mathrm{mol} \mathrm{L}^{-1}$ e relação solo: solução final de 1:100. Para a obtenção das doses de Zn e Cu foi utilizada solução padrão Merck® Certipur $1.000 \mathrm{mg} \mathrm{L}^{-1}$. A adsorção de $\mathrm{Cu}$ apresentou comportamento sigmoide (isoterma tipo $\mathrm{S}$ ), em solo sem dejeto (SD), e comportamento linear (isoterma tipo C), em solo com dejeto (CD). A capacidade máxima de adsorção (CMA) de $\mathrm{Cu}$ no solo SD foi de $3.021 \mathrm{mg} \mathrm{kg}^{-1}$. Para $\mathrm{Zn}$, todas as isotermas apresentaram comportamento linear. Não foi atingida a capacidade máxima de adsorção, no tratamento $\mathrm{CD}$, para $\mathrm{Cu}$ e $\mathrm{Zn}$. Esses resultados indicam que o Latossolo estudado apresenta alta capacidade para reter o $\mathrm{Cu}$ e $\mathrm{Zn}$ oriundos dos dejetos suínos.

Palavras-chave: isotermas, contaminação química, adubação orgânica.

\section{ABSTRACT}

\section{$\mathrm{Cu}$ and $\mathrm{Zn}$ adsorption in an Oxisol treated with pig slurry}

Cooper and zinc derived from pig slurry can accumulate in the soil, posing risks to human and animal health. The objective of this study was to determine the adsorption capacity and the competition of $\mathrm{Cu}$ and $\mathrm{Zn}$ in Oxisols that received continued applications of $200 \mathrm{~m}^{3} \mathrm{ha}^{-1} \mathrm{year}^{-1}$ of pig slurry for seven years and to compare with soil under natural conditions. To evaluate the adsorption, $\mathrm{Cu}$ and $\mathrm{Zn}$ solutions were applied to the soil at concentrations of 0 , 25, 50, 100, 150, 200, 400, $1.200 \mu \mathrm{mol} \mathrm{L}{ }^{-1}$; soil:solution ratio of 1:100.Merck Certipur standard solution 1.000 $\mathrm{mg} \mathrm{L}^{-1}$ was utilized to construct the levels of $\mathrm{Zn}$ and $\mathrm{Cu}$. The adsorption of $\mathrm{Cu}$ showed a sigmoid behavior (S-type isotherm) in soil with no slurry application and linear behavior (C-type isotherm) in soil with pig slurry. The maximum adsorption of $\mathrm{Cu}$ in soil without pig slurry was $3,021 \mathrm{mg} \mathrm{kg}^{-1}$. For $\mathrm{Zn}$, all the isotherms showed linear behavior. The maximum adsorption capacity of $\mathrm{Cu}$ and $\mathrm{Zn}$ was not reached in soil with slurry. These results show that the studied Oxisol has a high capacity to retain $\mathrm{Zn}$ and $\mathrm{Cu}$ from pig slurry.

Key words:chemical contamination, isotherms, organic fertilization.

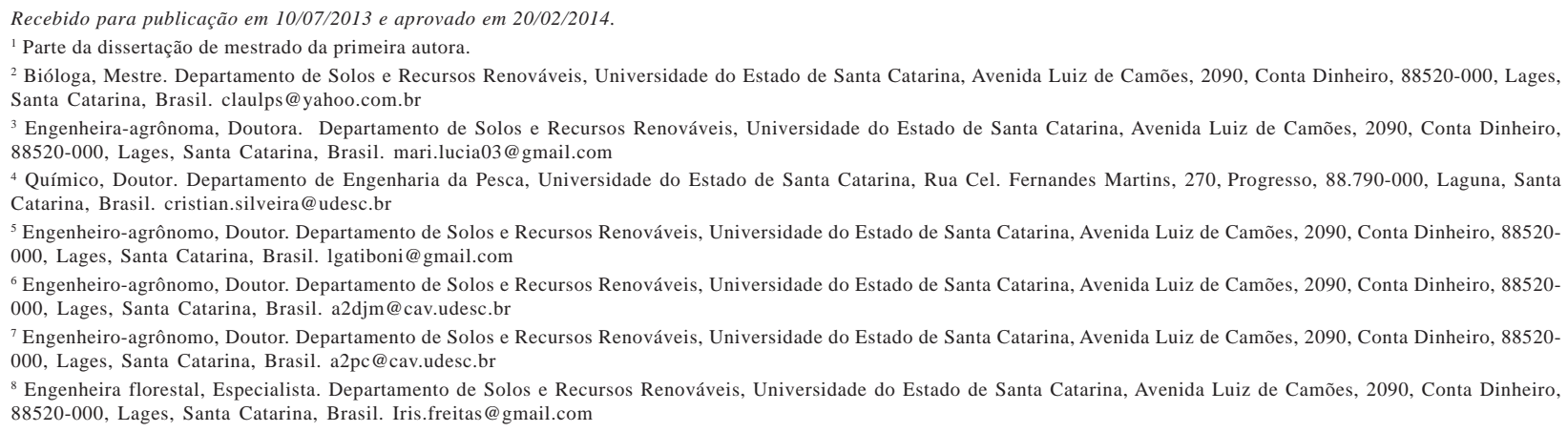




\section{INTRODUÇÃO}

Em criações de animais confinados são produzidos resíduos que podem ser utilizados como fertilizante. Diferentemente da adubação mineral, que pode ser formulada para cada cultura e solo, nos dejetos, os nutrientes apresentam-se em quantidades desproporcionais quanto à capacidade de absorção pelas plantas, podendo causar desequilíbrios químicos, físicos e biológicos no solo, como poluição das águas e redução da diversidade de plantas e de organismos do solo (Seganfredo, 2007).

Em virtude do fornecimento de rações altamente concentradas, os dejetos suínos apresentam teores elevados de $\mathrm{Cu}$ e $\mathrm{Zn}$. Teores em torno de 5 a $6 \mathrm{mg} \mathrm{kg}^{-1}$ de $\mathrm{Cu}$ e de 15 a $50 \mathrm{mg} \mathrm{kg}^{-1}$ de $\mathrm{Zn}$ são essenciais para o metabolismo dos suínos. A adição de 100 a $250 \mathrm{mg} \mathrm{kg}^{-1}$ de sulfato de cobre e de até $3000 \mathrm{mg} \mathrm{kg}^{-1}$ de óxido de zinco à dieta estimula o crescimento desses animais, sem causar efeitos tóxicos (NCR, 1998). Contudo, cerca de 90 $\%$ do $\mathrm{Cu}$ e $\mathrm{Zn}$ adicionados à ração são excretados nas fezes (Schmidt et al., 2007).

$\mathrm{O} \mathrm{Cu}$ e $\mathrm{Zn}$ excretados dos suínos podem acumularse no solo e propiciar a entrada em excesso desses elementos na cadeia alimentar, oferecendo riscos à saúde humana e à animal. Segundo López Alonso et al. (2000), altas concentrações de $\mathrm{Cu}$ têm sido detectadas em bovinos, na região agrícola da Galícia, noroeste da Espanha, principalmente onde existem criações intensivas de suínos. Estes autores encontraram mais de $20 \%$ do gado com concentrações de $\mathrm{Cu}$, no fígado, que excediam a concentração potencialmente tóxica de $150 \mathrm{mg} \mathrm{kg}^{-1}$, nas áreas de maior densidade de suínos. $\mathrm{O} \mathrm{Cu}$ e $\mathrm{Zn}$ acumulados no solo por aplicação de dejetos suínos também podem contaminar as águas superficiais, por meio do escoamento superficial, conforme estudo realizado por Girotto (2007). Esse autor encontrou transferência por escoamento superficial de 7,8, 4,9 e 2,1\%, do $\mathrm{Cu}$-total aplicado, e de 33, 4,4 e 2,5\% do Zn-total, nas doses de 20, 40 e $80 \mathrm{~m}^{3} \mathrm{ha}^{-1} \mathrm{ano}^{-1}$ de dejeto, respectivamente.

Em altas concentrações, o $\mathrm{Cu}$ e o $\mathrm{Zn}$ podem ser tóxicos às plantas. Segundo Gupta (2001), o sintoma mais comum de toxidade de $\mathrm{Cu}$ é a redução do crescimento das plantas. Para o Zn, Dudka et al. (1994) observaram que a dose $1.000 \mathrm{mg} \mathrm{kg}^{-1}$ reduziu significativamente a produção do trigo (40\%) e que com a aplicação de 1.500 $\mathrm{mg} \mathrm{kg}^{-1}$ não houve produção de grãos. Cunha et al. (2008) encontraram níveis tóxicos de Zn entre 74,1 e 110,7 mg $\mathrm{kg}^{-1}$ para a cultura do milho.

A exposição a concentrações elevadas de $\mathrm{Cu}$ e $\mathrm{Zn}$ pode causar danos ao organismo de animais e de humanos. Complicações nos sistemas hepático e renal foram observadas com a ingestão de Cu (ATSDR, 2004). A intoxicação crônica pela ingestão de $\mathrm{Zn}$ pode causar ane- mia, danos pancreáticos e diminuição do HDL no sangue (ATSDR, 2005).

Quanto à disponibilidade do $\mathrm{Cu}$ e $\mathrm{Zn}$ provenientes dos dejetos suínos no perfil do solo, Saviozzi et al. (1997) encontraram aumento nos teores totais de $\mathrm{Cu}$ e Zn nos solos com aplicação desses dejetos, porém, a maior parte desses elementos ligou-se à fração não lábil do solo. Portanto, apesar do aumento dos teores $\mathrm{Cu}$ e Zn no solo, estando esses elementos na forma residual, eles não estarão disponíveis para serem absorvidos pelas plantas. L'Herrox et al. (1997) obtiveram maior incremento de $\mathrm{Cu}$ proveniente dos dejetos na fração orgânica $(65 \%)$ e, para o $\mathrm{Zn}$, o incremento maior foi nos óxidos de ferro (40\%). Porém, para melhor entendimento da disponibilidade dos metais, é necessário o conhecimento dos processos que regem o transporte e a retenção dos metais entre as superfícies sólidas e líquidas do solo.

A capacidade que o solo tem de adsorver o $\mathrm{Cu}$ e o $\mathrm{Zn}$ adicionados por via de dejetos é finita. Quando a capacidade máxima de adsorção (CMA) é ultrapassada, o Cu e o Zn solúveis aumentam. O processo de adsorção determina a quantidade de nutrientes, metais, pesticidas e outros compostos orgânicos que são retidos na superfície do solo e, portanto, é um dos processos primários que afetam o transporte de nutrientes e contaminantes no solo (Sparks, 1995). Os estudos de adsorção podem ser realizados por meio de isotermas de adsorção, que dão indícios sobre a capacidade dos metais de adsorverem-se no solo (Hinz, 2001). No entanto, outros mecanismos podem estar envolvidos nos fenômenos de sorção, como a precipitação, a complexação, a fixação etc. (Bradl, 2004).

A presença de elementos competidores também pode afetar a adsorção. Moreira \& Alleoni (2010), avaliaram a adsorção competitiva de $\mathrm{Cd}, \mathrm{Ni}, \mathrm{Zn}$ e $\mathrm{Cu}$, em solos de São Paulo, e perceberam que a competição diminuiu a CMA dos metais. A CMA também pode variar de acordo com o tipo de solo. Solos argilosos ou bastante intemperizados e, por isso, com maior número de sítios de adsorção, tendem a apresentar maior CMA.

O objetivo deste trabalho foi determinar a capacidade de adsorção e a competição de $\mathrm{Cu}$ e $\mathrm{Zn}$, num solo que vem recebendo aplicações continuadas de dejetos suínos, e comparar sua adsorção com a de solo que não recebeu dejetos.

\section{MATERIAL E MÉTODOS}

Os solos utilizados neste estudo são oriundos de áreas vizinhas e contíguas e pertencem à mesma classe, Latossolo Vermelho distroférrico. O que os diferencia é que um recebeu dejetos suínos, durante sete anos, com 
taxa de aplicação de $200 \mathrm{~m}^{3} \mathrm{ha}^{-1} \mathrm{ano}^{-1}$, sob cultivo anual, ou seja, tratamento com dejeto (CD), enquanto o outro nunca recebeu dejetos, sendo mantido em condições naturais (mata nativa), tratamento sem dejetos (SD). A área, denominada CD, faz parte de experimento implantado em 2001, no município de Campos Novos, a cerca de $20 \mathrm{~km}$ da cidade, nas coordenadas geográficas $27^{\circ} 23^{\prime} 33^{\prime \prime} \mathrm{S}$, e $51^{\circ} 21^{\prime} 48^{\prime \prime} \mathrm{O}$, em altitude de $862 \mathrm{~m}$. A área da parcela no tratamento CD era de 75,6 $\mathrm{m}^{2}$ (12 $\mathrm{m}$ x 6,3 m), cultivada anualmente com a sucessão milho (Zea mays L.) e aveia preta (Avena strigosa L.), no sistema de plantio direto. No quarto ano, a aveia foi substituída pelo nabo forrageiro (Raphanus sativus L.). O dejeto foi semi-estabilizado, sendo recolhido diretamente da esterqueira a céu aberto, alimentada em fluxo contínuo, sendo o resíduo armazenado por cerca de quatro meses.

Ressalta-se que $50 \mathrm{~m}^{3}$ ha $^{-1}$ ano $^{-1}$ é a taxa de aplicação permitida de dejetos líquidos de suínos ao solo (FATMA, 2004). O motivo que levou à decisão por uma dose máxima alta $\left(200 \mathrm{~m}^{3} \mathrm{ha}^{-1}\right)$ foi a possibilidade de potencializar e acelerar o surgimento de possíveis problemas de poluição, como os excessos de N, P, Cu e Zn, bem como o surgimento de possíveis estados de saturação de atributos como adsorção de $\mathrm{P}, \mathrm{Cu}, \mathrm{Zn}$ e dos teores de $\mathrm{C}$ orgânico e de $\mathrm{N}$ total no solo.

O solo foi coletado, dez meses após a última aplicação de dejetos suínos, na camada de $0-10 \mathrm{~cm}$, sendo que, no momento da coleta, a aveia preta era a cultura no local. Foram utilizadas três repetições, em que cada amostra foi composta de oito amostras simples, coletadas com trado holandês. As amostras foram secadas ao ar, moídas e passadas por peneira com abertura de 2,0 mm de malha.

As Tabelas 1, 2 e 3 apresentam características químicas e a argila do Latossolo Vermelho distroférrico, os teores de macronutrientes e de micronutrientes determinados no tratamento $\mathrm{CD}$ e de macronutrientes e de micronutrientes determinados no dejeto suíno, respectivamente.

Para avaliar o comportamento adsortivo do $\mathrm{Cu}$ e $\mathrm{Zn}$ nos tratamentos $\mathrm{SD}$ e $\mathrm{CD}$, foram preparadas suspensões de TFSA dos solos, em solução salina de $\mathrm{Ca}\left(\mathrm{NO}_{3}\right)_{2}$, com força iônica igual a $15 \mathrm{mmol} \mathrm{L}^{-1}$, na relação $1: 68(0,5 \mathrm{~g}$ de solo $+34 \mathrm{~mL}$ de solução salina). $\mathrm{O}$ pH da suspensão foi ajustado para 5,5 $( \pm 0,2)$, com soluções de $\mathrm{Ca}(\mathrm{OH})_{2} 0,01$ mol L-1, ou de $\mathrm{HNO}_{3}$ 0,01 mol L-1. As amostras (solo + solução salina) foram submetidas a ciclos de agitação (12 horas de agitação e 12 horas de repouso), até a obtenção e estabilização do pH desejado. O pH 5,5 foi o escolhido, pois, segundo Agbenin \& Olojo (2004), a adsorção de $\mathrm{Cu}$ aumenta acentuadamente com aumento do $\mathrm{pH}$ 4,0 a 5,5, enquanto que o aumento da adsorção de Zn ocorre com o aumento do pH 5,3 a 6,8. Após a estabilização do pH e antes de se adicionarem as doses de $\mathrm{Cu}$ e $\mathrm{Zn}$, foram realizadas leituras preliminares de $\mathrm{Cu}$ e $\mathrm{Zn}$, na solução de equilíbrio, por espectrofotometria de absorção atômica, nas amostras CD. Não foi observado $\mathrm{Cu}$ na solução de equilíbrio, porém, em média, foram encontrados $0,13 \mathrm{mg} \mathrm{kg}^{-1} \mathrm{de}$ $\mathrm{Zn}$ em cada amostra. Para evitar que os teores de Zn solúvel presentes nas amostras de solo CD interferissem nos

Tabela 1. Características gerais do Latossolo Vermelho distroférrico em 2001, antes do início da aplicação continuada de dejetos suínos, camada de 0 - $20 \mathrm{~cm}$ (Almeida et al., 2003) e teores de Cu e Zn no solo segundo Silva (2009)

\begin{tabular}{|c|c|c|c|c|c|c|c|c|c|c|c|c|}
\hline \multirow[t]{2}{*}{ Prof } & pH & \multirow[t]{2}{*}{ MO } & \multirow[t]{2}{*}{$\mathbf{C}$} & \multirow[t]{3}{*}{ SB } & \multirow[t]{2}{*}{ Al } & CTC & \multirow[t]{2}{*}{$\mathbf{V}$} & \multirow[t]{2}{*}{ Arg } & $\mathbf{C u}$ & $\mathbf{Z n}$ & $\mathbf{C u}$ & $\mathbf{Z n}^{*}$ \\
\hline & $\mathrm{H}_{2} \mathrm{O}$ & & & & & \multirow[t]{2}{*}{ pH 7} & & & \multicolumn{2}{|c|}{ Extraível } & \multicolumn{2}{|c|}{ Semitotal } \\
\hline \multicolumn{2}{|l|}{$\mathrm{cm}$} & \multicolumn{2}{|c|}{$\mathrm{g} \mathrm{kg}^{-1}$} & & $\mathrm{cmol}_{\mathrm{c}} \mathrm{kg}^{-1}$ & & $\%$ & $\mathrm{~g} \mathrm{~kg}^{-1}$ & \multicolumn{4}{|c|}{$\mathrm{mg} \mathrm{kg}^{-1}$} \\
\hline $0-20$ & 4,6 & 51,7 & 29,9 & 2,4 & 3,4 & 20,5 & 12 & 640 & 6,2 & 1,4 & 253 & 103 \\
\hline
\end{tabular}

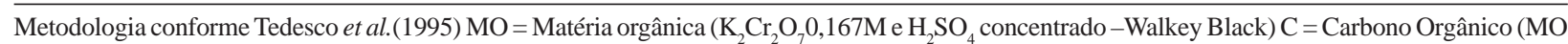

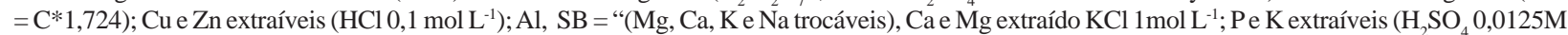
+ $\mathrm{HCl}$ 0,05M -Mehlich 1). Metodologia conforme EMBRAPA, (1997): CTC a pH 7,0; Arg = Teor de argila (método da pipeta). * Teor de Cu e Zn na camada de 0-10 cm obtidos pelo método USEPA 3050B (dados do próprio autor).

Tabela 2. Teores de macro e micronutrientes encontrados no solo com dejetos suínos, na camada de 0-10 e 10-20 cm após 7 anos de aplicação de dejetos suínos na dose $200 \mathrm{~m}^{3} \mathrm{ha}^{-1} \mathrm{ano}^{-1}$ (Silva, 2009)

\begin{tabular}{|c|c|c|c|c|c|c|c|c|c|c|c|c|}
\hline \multirow[t]{2}{*}{ Prof } & $\mathbf{C}$ & $\mathbf{N}$ & pH & Al & $\mathrm{Ca}$ & Mg & $\mathbf{P}$ & $\mathbf{K}$ & $\mathrm{Cu}$ & $\mathbf{Z n}$ & $\mathrm{Cu}$ & $\mathbf{Z n}$ \\
\hline & org & total & \multirow[t]{2}{*}{$\mathrm{H}_{2} \mathrm{O}$} & & & & & & \multicolumn{2}{|c|}{ extraível } & \multicolumn{2}{|c|}{ Semitotal* } \\
\hline $\mathrm{cm}$ & \multicolumn{2}{|c|}{$\mathrm{kg} \mathrm{m}^{-3}$} & & \multicolumn{3}{|c|}{ cmolcdm $^{-3}$} & \multicolumn{6}{|c|}{$\mathrm{mg} \mathrm{kg}^{-1}$} \\
\hline $0-10$ & 52 & 3,3 & 5,5 & 0,0 & 10,7 & 5,1 & 345 & 1524 & 14 & 46 & 265 & 117 \\
\hline $10-20$ & 32 & 1,9 & 5,5 & 0,0 & 6,9 & 2,5 & 15 & 1041 & 7,9 & 7,2 & - & - \\
\hline
\end{tabular}

Metodologia conforme Tedesco et al.(1995): C orgânico $\left(\mathrm{K}_{2} \mathrm{Cr}_{2} \mathrm{O}_{7} 0,167 \mathrm{M} \mathrm{e} \mathrm{H}_{2} \mathrm{SO}_{4}\right.$ concentrado -Walkey Black); $\mathrm{N}$ total $\left(\mathrm{H}_{2} \mathrm{SO}_{4}\right.$ concentrado - Kjeldahl) $\mathrm{Al}, \mathrm{Mg}, \mathrm{Ca}$ trocáveis ( $\left.\mathrm{KCl} 1 \mathrm{~mol} \mathrm{~L}^{-1}\right) ; \mathrm{P}$ e K extraíveis $\left(\mathrm{H}_{2} \mathrm{SO}_{4} 0,0125 \mathrm{M}+\mathrm{HCl}\right.$ 0,05M -Mehlich 1); Cu e $\mathrm{Zn}$ extraíveis ( $\mathrm{HCl}$

0,1 $\mathrm{mol} \mathrm{L}^{-1}$ ). * Teor de $\mathrm{Cu}$ e $\mathrm{Zn}$ na camada de 0-10 $\mathrm{cm}$ obtidos pelo método USEPA 3050B (dados do próprio autor). 
Tabela 3. Valores de pH e teores de macro e micronutrientes no dejeto líquido (base úmida) utilizado nas aplicações anuais (Silva, 2009; Costa, 2011)

\begin{tabular}{|c|c|c|c|c|c|c|c|c|c|c|c|c|}
\hline \multirow[t]{2}{*}{ Aplicação } & \multirow[t]{2}{*}{ pH } & MS & C-org & $\mathbf{N}$ & $\mathbf{P}$ & $\mathbf{K}$ & $\mathbf{C a}$ & Mg & $\mathrm{Cu}$ & $\mathbf{Z n}$ & $\mathbf{F e}$ & Mn \\
\hline & & \multicolumn{7}{|c|}{$\mathrm{kg} \mathrm{m}^{-3}$} & \multicolumn{4}{|c|}{$\mathrm{g} \mathrm{m}^{-3}$} \\
\hline $10 / 2001$ & 6,7 & 66 & 23,8 & 3,4 & 1,4 & 1,2 & 3,3 & 1,1 & 1,2 & 8,0 & 11,9 & 5,2 \\
\hline $11 / 2002$ & 7,1 & 26 & 11,2 & 2,6 & 1,0 & 1,2 & 1,8 & 0,7 & ND & ND & ND & ND \\
\hline $10 / 2003$ & 6,9 & 32 & 12,8 & 2,6 & 1,1 & 1,3 & 2,1 & 0,9 & ND & ND & ND & ND \\
\hline $10 / 2004$ & 7,3 & 43 & 16,6 & 3,7 & 1,4 & 1,5 & 2,8 & 1,2 & ND & ND & ND & ND \\
\hline $10 / 2005$ & 7,8 & 56 & 23,0 & 3,2 & 1,5 & 1,1 & 1,8 & 0,9 & 8,8 & 3,2 & ND & ND \\
\hline $10 / 2006$ & 7,0 & 114 & 37,8 & 4,6 & 2,8 & 1,7 & 1,7 & 0,9 & 3,4 & 4,6 & 9 & 12,8 \\
\hline $10 / 2007$ & 7,3 & 55 & 21,3 & 2,7 & 1,8 & 1,1 & 1,5 & 0,8 & 4,1 & 7,8 & 2,7 & 9,6 \\
\hline Média & 7,2 & 56,0 & 20,9 & 3,3 & 1,6 & 1,3 & 2,1 & 0,9 & 4,4 & 5,9 & 7,9 & 9,2 \\
\hline
\end{tabular}

Metodologia conforme Tedesco et al, (1995): C orgânico $\left(\mathrm{K}_{2} \mathrm{Cr}_{2} \mathrm{O}_{7} 0,167 \mathrm{M} \mathrm{e} \mathrm{H}_{2} \mathrm{SO}_{4}\right.$ concentrado -Walkey Black); $\mathrm{N}$ total $\left(\mathrm{H}_{2} \mathrm{SO}_{4}\right.$ concentrado Kjeldahl); Pe Kextraíveis $\left(\mathrm{H}_{2} \mathrm{SO}_{4}\right.$ 0,0125M + $\mathrm{HCl}$ 0,05M -Mehlich 1); Mn, Mg, Ca trocáveis ( $\left.\mathrm{KCl} 1 \mathrm{~mol} \mathrm{~L}^{-1}\right)$; Cu, Fe, Mn e Zn extraíveis $\left(\mathrm{HCl}_{0}, 1 \mathrm{~mol} \mathrm{~L}^{-1}\right)$.

resultados, foram realizadas extrações desse elemento, utilizando-se o $\mathrm{Ca}\left(\mathrm{NO}_{3}\right)_{2} 15 \mathrm{mmol} \mathrm{L}^{-1}$, até a completa remoção do $\mathrm{Zn}$.

Após essa etapa, as amostras receberam $16 \mathrm{~mL}$ de solução, contendo $\mathrm{Cu}$ ou $\mathrm{Zn}$, nas seguintes concentrações: $0,25,50,100,150,200,400,1.200 \mu \mathrm{mol} \mathrm{L}^{-1}$, equivalentes a 0,$0 ; 1,5 ; 3,0 ; 6,0 ; 9,5 ; 13,0 ; 25,0 ; 76,0 \mathrm{mg} \mathrm{L}^{-1}$, para $\mathrm{Cu}$, e 0,$0 ; 1,6 ; 3,0 ; 6,5 ; 10,0 ; 13,0 ; 26,0 ; 78,0 \mathrm{mg} \mathrm{L}^{-}$ ${ }^{1}$, para $\mathrm{Zn}$. Para obtenção das doses de $\mathrm{Zn}$ e $\mathrm{Cu}$ foram utilizadas soluções padrão Merck Certipur $1.000 \mathrm{mg} \mathrm{L}$ ${ }^{1}$. A relação solo: solução final foi de 1:100. Após a estabilização do pH e adição das alíquotas contendo os metais, as amostras foram incubadas por 72 horas, com agitação alternada (120 rpm), sendo 12 horas de agitação e 12 horas de repouso. Por fim, as suspensões foram centrifugadas e a dosagem de $\mathrm{Cu}$ e $\mathrm{Zn}$ foi realizada por espectrofotometria de absorção atômica.

$\mathrm{O}$ efeito competitivo do $\mathrm{Cu}$ e $\mathrm{Zn}$ nos tratamentos SD e CD foi avaliado, utilizando-se as mesmas condições experimentais usadas para determinar a adsorção dos elementos isolados, porém, as soluções $\mathrm{Cu}$ e $\mathrm{Zn}$ foram adicionadas simultaneamente nas amostras, nas mesmas concentrações mencionadas acima.

A quantidade adsorvida foi calculada, aplicando-se as seguintes equações: $\mathrm{q}=\{(\mathrm{Ci}-\mathrm{Ce}) *[(\mathrm{~V} 1+\mathrm{V} 2) / \mathrm{Ms}]\}$; Madc $=\left\{\left(\mathrm{Ci}^{*}[(\mathrm{~V} 1+\mathrm{V} 2) / \mathrm{Ms}]\right\}\right.$ e $\% \mathrm{q}=(\mathrm{q} / \mathrm{Madc}) * 100$, sendo q, o $\mathrm{Cu}$ ou $\mathrm{Zn}$ adsorvido, em $\mathrm{mg} \mathrm{kg}^{-1}$ de solo; $\mathrm{Ce}$, a concentração de equilíbrio depois de 72 horas, em mg $\mathrm{L}^{-1}$; Ms, a massa do solo, em g; Madc, a quantidade do metal, adicionado, em $\mathrm{mg} \mathrm{L}^{-1}$ de solo; $\mathrm{Ci}$, a concentração inicial de $\mathrm{Cu}$ e $\mathrm{Zn}$, em mg L $\mathrm{L}^{1} ; \mathrm{V}_{1}$, o volume, em mL, da solução padrão de $\mathrm{Cu}$ para se atingir as concentrações de 0,$0 ; 1,5 ; 3,0 ; 6,0 ; 9,5 ; 13,0 ; 25,0 ; 76,0 \mathrm{mg} \mathrm{L}^{-1}$, ou da solução de $\mathrm{Zn}$ para se atingirem as concentrações de 0,$0 ; 1,6 ; 3,0 ; 6,5 ; 10,0 ; 13,0 ; 26,0 ; 78,0 \mathrm{mg} \mathrm{L}^{-1} ; \mathrm{V}_{2}$, o volume, em mL, de solução de $\mathrm{Ca}\left(\mathrm{NO}_{3}\right)_{2}$; e \% q, a percentagem de $\mathrm{Cu}$ e $\mathrm{Zn}$ adsorvido pelo solo.
Para identificação do tipo de isoterma, foram relacionados, num gráfico, a adsorção (q) em função da concentração de equilíbrio (Ce) Sparks (1995), ou seja, a concentração do metal determinada após as 72 horas de incubação e ajustados os modelos convenientes, no caso, sigmoides (S) ou lineares (C), de acordo com classificação proposta por Giles et al. (1974). Apenas as isotermas do tipo C, tiveram suas inclinações e interceptos comparados. A capacidade máxima de adsorção (CMA) foi calculada somente nos casos de ajuste de isotermas do tipo $\mathrm{S}$, nos quais a CMA é o valor obtido quando a adsorção se estabiliza, formando um patamar. Nas situações em que se ajustaram isotermas do tipo $\mathrm{C}$ não foi possível calcular a CMA, pois, neste caso, não há estabilização da adsorção.

Os resultados foram submetidos a análises de variância e de regressão, de acordo com o delineamento inteiramente casualizado, em parcelas subdivididas. As parcelas foram constituídas pelos tratamentos SD e CD, enquanto as subparcelas foram formadas pelas combinações dos níveis dos fatores sistema competitivo e doses de $\mathrm{Cu}$ e $\mathrm{Zn}$ adicionadas. Os efeitos simples dos fatores adsorção de $\mathrm{Cu}$ e de Zn e sistema competitivo, na presença de interação, foram desdobrados e comparados pelo teste de Tukey. Para se atender às pressuposições teóricas do referido teste, aplicou-se a transformação logarítmica das variáveis adsorção de $\mathrm{Cu}$ e de $\mathrm{Zn}$, conforme sugerido pela análise descritiva dos dados. No entanto, os resultados são apresentados na escala original. Para todos os testes efetuados, foi considerado o nível mínimo de significância de $5 \%$. Todas as análises foram conduzidas usando-se o software SAS® (StatisticalAnalysis System, 2003).

\section{RESULTADOS E DISCUSSÃO}

Em competição com o $\mathrm{Zn}$, a adsorção média de $\mathrm{Cu}$ foi maior no tratamento CD (Tabela 4) e isso se deve, 
provavelmente, à existência de maior número de sítios em coloides orgânicos, com os quais o $\mathrm{Cu}$ tem grande afinidade (Han et al., 2000; Nascimento \& Fontes, 2004). Para o $\mathrm{Cu}$ isolado, a adsorção foi maior no tratamento SD, porém, o sistema competitivo expressa uma avaliação mais realista do comportamento dos metais no solo (Moreira, 2004). No solo SD, não houve diferença entre a adsorção do $\mathrm{Cu}$ isolado e a do $\mathrm{Cu}$ em competição, mostrando que o $\mathrm{Zn}$ não restringiu a adsorção de $\mathrm{Cu}$. No tratamento $\mathrm{CD}$, a adsorção do $\mathrm{Cu}$, competindo, foi maior. Portanto, em sistema competitivo, o $\mathrm{Cu}$ parece ter preferência pelas cargas. Os metais mais eletronegativos formam ligações iônicas, com maior caráter de covalência, com átomos de oxigênio da superfície dos coloides do solo e, portanto, mais fortes. Para alguns metais divalentes, a preferência pelos constituintes do solo pode ser $\mathrm{Cu}>\mathrm{Ni}>\mathrm{Co}>\mathrm{Pb}>\mathrm{Cd}>\mathrm{Zn}$ $>\mathrm{Mg}>\mathrm{Sr}$ (Mcbride, 1994). Moreira \& Alleoni (2010) avaliaram a adsorção de metais em 14 solos representativos da região de São Paulo e verificaram que a sequência de afinidade dos metais mais comumente encontrada nos solos estudados foi $\mathrm{Cu}>\mathrm{Zn}>\mathrm{Ni}>\mathrm{Cd}$. Dos 14 solos analisados, em apenas 4 (Arenic Hapludult, Arenic Hapludalf, Typic Quartzipsamment, Typic Qystrochept), não foi encontrado o $\mathrm{Cu}$ como o elemento de maior afinidade. Esses solos apresentavam textura mais arenosa, baixa CTC, baixos teores de carbono orgânico, em comparação com os outros solos analisados.

A média de adsorção para $\mathrm{Zn}$, tanto isolado quanto em competição, foi maior no tratamento SD (Tabela 4), indicando ausência de efeito da adição de dejetos sobre a adsorção de Zn. Como, neste solo, o teor de carbono orgânico tende a ser menor, espera-se que a adsorção dependa mais de sítios de ligação de coloides inorgânicos (i.e. óxidos, hidróxidos e oxihidróxidos de $\mathrm{Fe}$ e Al, principalmente). Outros autores, realizando extrações sequen-

Tabela 4. Média das percentagens de adsorção para as concentrações $0,25,50,100,150,200,400,1200 \mathrm{mmol} \mathrm{L}^{-1} \mathrm{de} \mathrm{Cu}$ e $\mathrm{Zn}$ isolado e competindo nos tratamentos sem dejeto (SD) e com dejeto (CD)

\begin{tabular}{lcc}
\hline & \multicolumn{2}{c}{ Adsorção \% } \\
\cline { 2 - 3 } & \multicolumn{2}{c}{ Cu } \\
\cline { 2 - 3 } & Competindo & Isolado \\
\hline $\mathrm{SD}$ & $16,45 \mathrm{Ba}^{*}$ & $15,71 \mathrm{Aa}$ \\
$\mathrm{CD}$ & $20,51 \mathrm{Aa}$ & $13,54 \mathrm{Bb}$ \\
\hline & \multicolumn{3}{c}{$\mathbf{Z n}$} \\
\hline $\mathrm{SD}$ & $19,56 \mathrm{Ab}$ & $27,17 \mathrm{Aa}$ \\
$\mathrm{CD}$ & $12,56 \mathrm{Bb}$ & $19,00 \mathrm{Ba}$ \\
\hline
\end{tabular}

Letras maiúsculas comparam a \% adsorvida de $\mathrm{Cu}$ e $\mathrm{Zn}$ nas colunas (tratamento SD vs. CD) e letras minúsculas comparam a \% adsorvida nas linhas (sistema competitivo).

* Significativo a $5 \%$ pelo teste de Tukey. ciais, observaram que a fração mineral é a mais significativa na adsorção de Zn (Silveira et al., 2008; Girotto et al., 2010). A média de adsorção de Zn foi maior em sistema isolado que em competitivo, indicando o efeito supressor do $\mathrm{Cu}$ em relação ao Zn. A presença de íons competidores geralmente altera a adsorção dos metais avaliados individualmente. Pierangeli et al. (2007) estudaram o comportamento competitivo de $\mathrm{Cu}, \mathrm{Cd}$ e $\mathrm{Pb}$ em concentrações que variaram de 0 a $47 \mathrm{mg} \mathrm{kg}^{-1}$ e obtiveram redução da adsorção dos três elementos, quando eles se encontravam simultaneamente no sistema.

Com base nas isotermas de adsorção (Figura 1a), observa-se que, no tratamento SD, a adsorção de $\mathrm{Cu}$ apresentou comportamento sigmoide, característico de isoterma tipo $\mathrm{S}$ e, no tratamento $\mathrm{CD}$, apresentou comportamento linear, característico de isoterma tipo C, conforme classificação proposta por Giles et al. (1974).

O ponto de inflexão das isotermas tipo S (Figura 1a) ocorre por causa de duas situações: a) em baixas concentrações a superfície tem uma pequena afinidade pelo elemento, a qual aumenta com o teor de adsorbato da solução de equilíbrio (Sparks, 1995); b) pela presença de compostos ligantes solúveis, pois somente após a saturação do ligante solúvel a adsorção passará a ocorrer normalmente nos sítios dos coloides (Limousin et al., 2007; Sposito 2008). Acredita-se que, no tratamento CD, os compostos orgânicos complexaram íons da solução, que atuariam como ligantes; a isoterma passou do tipo $\mathrm{S}$ para o tipo C (até a concentração Ce testada) e, ainda, a adsorção diminuiu de $3.021 \mathrm{mg} \mathrm{kg}^{-1}$ para $1.977 \mathrm{mg} \mathrm{kg}^{-1}$ na Ce de 50 $\mathrm{mg} \mathrm{L}^{-1}$.

Para $\mathrm{Cu}$, no tratamento SD (Figura 1a), após a concentração de $30 \mathrm{mg} \mathrm{L}^{-1}$, a adsorção alcançou um patamar de equilíbrio das reações de adsorção. Esse equilíbrio pode ser devido à saturação dos sítios de adsorção, indicando que o solo atingiu a CMA. A CMA de Cu para solo $\mathrm{SD}$ foi de $3.021 \mathrm{mg} \mathrm{kg}^{-1}$. O solo apresentou elevada CMA, comparada com a de Latossolos obtida em outros trabalhos. Nascimento \& Fontes (2004) encontraram valores de CMA entre 1.008 e $2.008 \mathrm{mg} \mathrm{kg}^{-1}$, em Latossolos de Minas Gerais, com valores de $\mathrm{Cu}$ na $\mathrm{Ce}$ entre $0-140$ $\mathrm{mg} \mathrm{L}^{-1}$ à $\mathrm{pH}$ 5,5. Pierangeli et al. (2007) encontraram $1.659 \mathrm{mg} \mathrm{kg}^{-1}$, em Latossolo Vermelho, nas concentrações entre 0 e $48 \mathrm{mg} \mathrm{L}^{-1}$, em pH 5,5.

Não foi possível calcular a CMA de Cu para $\mathrm{CD}$, uma vez que a adsorção não alcançou um patamar de estabilização. A estabilização deverá ocorrer em concentrações de $\mathrm{Cu}$ acima das testadas neste experimento. A presença de estabilização da adsorção de $\mathrm{Cu}$ para SD e a ausência desta para CD podem ser indícios de aumento da CMA em tratamento $\mathrm{CD}$, pois é possível que a aplicação do dejeto aumente o número de grupamentos funcionais da matéria orgânica. Situação semelhante foi obtida por Paulín et 
al. (2006), avaliando o efeito do lodo de esgoto na sorção de $\mathrm{Cu}$ e $\mathrm{Cd}$. No solo sem lodo de esgoto foi alcançada a CMA de $\mathrm{Cu}$ e, no solo com aplicação de lodo, ela foi obtida na Ce entre 0 e $400 \mathrm{mg} \mathrm{L}^{-1}$, isoterma linear (tipo C), lembrando-se que, neste estudo, o intervalo obtido na $\mathrm{Ce}$ foi de 0 a $60 \mathrm{mg} \mathrm{L}^{-1}$ de $\mathrm{Cu}$. Os autores relacionaram esse tipo de isoterma ao aumento da adsorção de $\mathrm{Cu}$, proporcionado pela adição da matéria orgânica. Moreira \& Alleoni (2010) também não alcançaram valores para a CMA, em Latossolo Vermelho (Ce entre 0 e $120 \mathrm{mg} \mathrm{L}$ $\left.{ }^{1}\right)$. Os autores atribuíram isso à alta capacidade de adsorção do solo estudado.

A adsorção de $\mathrm{Zn}$ aumentou, linearmente, com o aumento da concentração inicial desse elemento nos tratamentos SD e CD (figura 1b), formando isotermas do tipo C. As retas ajustadas apresentam inclinações diferentes e mesmo intercepto. Casagrande et al. (2008) também obtiveram isotermas do tipo $\mathrm{C}$, estudando solos altamente intemperizados (Anionic "Xanthic" Acrudox e Anionic "Rhodic" Acrudox), com quantidades crescentes de $\mathrm{Zn}$ $\left(0-80 \mathrm{mg} \mathrm{L}^{-1}\right)$, pH natural e força iônica constante. Segundo os autores, esse tipo de isoterma não era o esperado, pois mostra que a disponibilidade dos sítios de adsorção manteve-se constante, independentemente da concentração inicial de $\mathrm{Zn}$.

$\mathrm{Na}$ presença de competição, as curvas de adsorção do $\mathrm{Cu}$ nos tratamentos SD e CD mostraram-se na forma de isotermas do tipo C, lineares (Figura 2a). Não houve diferença entre o tratamento SD e CD. As retas apresentam inclinações iguais e mesmo intercepto, portanto, uma única equação serviu para representar as duas situações. Agbenin \& Olojo (2004) avaliaram a
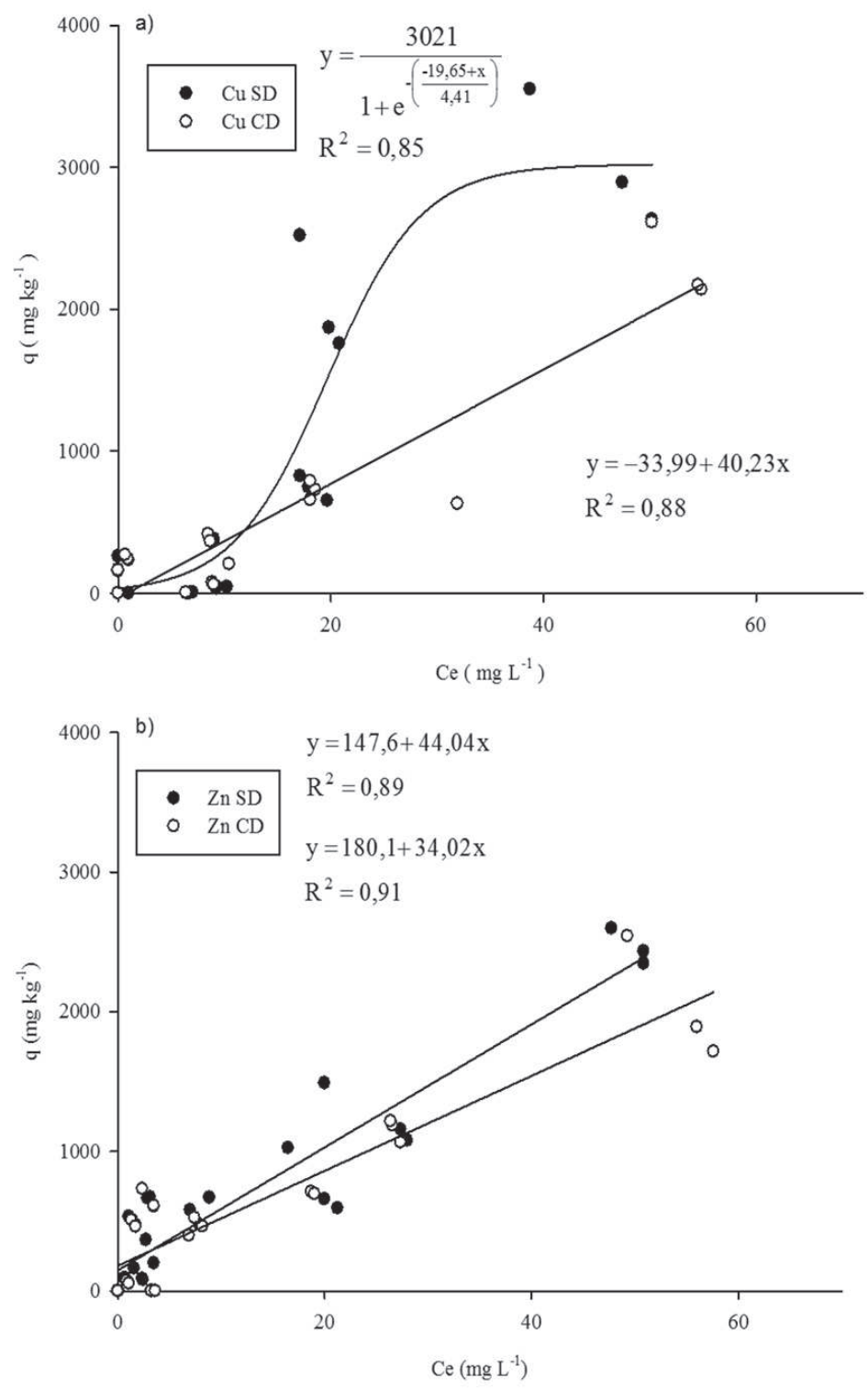

Figura 1. Isotermas de adsorção de $\mathrm{Cu}$ e $\mathrm{Zn}$ a pH 5,5 e força iônica $15 \mathrm{mmol} \mathrm{L} \mathrm{L}^{-1} \mathrm{em}$ Latossolo. Quantidade adsorvida (q) de $\mathrm{Cu}$ em função da concentração de equilíbrio (Ce). a) Adsorção de Cu no tratamento sem dejeto suíno (SD) e com dejeto (CD); b) Adsorção de Zn no tratamento sem dejeto suíno (SD) e com dejeto (CD).

Rev. Ceres, Viçosa, v. 61, n.6, p. 997-1005, nov/dez, 2014 
adsorção competitiva entre $\mathrm{Cu}$ e $\mathrm{Zn}$ em solo de horizonte Bt, utilizando concentrações de 10 a $400 \mathrm{mg} \mathrm{kg}^{-1}$, e obtiveram, para $\mathrm{Cu}$ isotermas do tipo $\mathrm{H}$ e, para $\mathrm{Zn}$, isoterma do tipo linear.

As inclinações e os interceptos da reta não diferiram estatisticamente, para o Zn isolado (Figura 1b) e em competição (Figura 2b), nos tratamentos $\mathrm{SD}$ e $\mathrm{CD}$. Quanto à comparação da adsorção do Zn, competindo, nos tratamentos SD e CD (Figura 2b), as retas também não diferiram.

Para $\mathrm{Cu}$, em particular, a aplicação de dejetos resultou em alterações, tanto no tipo de isoterma quanto na CMA. Estes dois fenômenos podem ser resultantes do aumento de compostos orgânicos solúveis, que apresentem habilidade de complexar o $\mathrm{Cu}$. A presença desses compostos acarretaria ampliação do ponto de inflexão da isoterma tipo $\mathrm{S}$, gerando uma isoterma tipo $\mathrm{C}$; neste caso, seria necessária a utilização de concentrações iniciais de $\mathrm{Cu}$ superiores àquelas adotadas neste trabalho, para que, após completa saturação desses compostos orgânicos, começassem a ocorrer, efetivamente, a adsorção e a saturação dos sítios de ligação presentes na fase sólida do solo. Comparando-se os teores de $\mathrm{Cu}$, extraível e semitotal, da camada $0-10 \mathrm{~cm}$, em solo que recebeu $200 \mathrm{~m}^{3} \mathrm{ha}^{-1} \mathrm{ano}^{-1}$ de dejetos suínos durante 7 anos (14 e $265 \mathrm{mg} \mathrm{kg}^{-1} \mathrm{de} \mathrm{Cu}$, respectivamente) (Tabela 2), e a CMA de $\mathrm{Cu}$, em solo $\mathrm{SD}$ (3.021 mg $\mathrm{kg}^{-1}$ ), na camada 0-10 cm, percebe-se que o solo ainda não atingiu a CMA. Para o Zn, a situação foi semelhante, já que o teor encontrado, no solo que recebeu dejetos, foi de 46 e $117 \mathrm{mg} \mathrm{kg}^{-1}$ de Zn extraível e semitotal, respectivamente (Tabela 3 ), e, na isoterma,
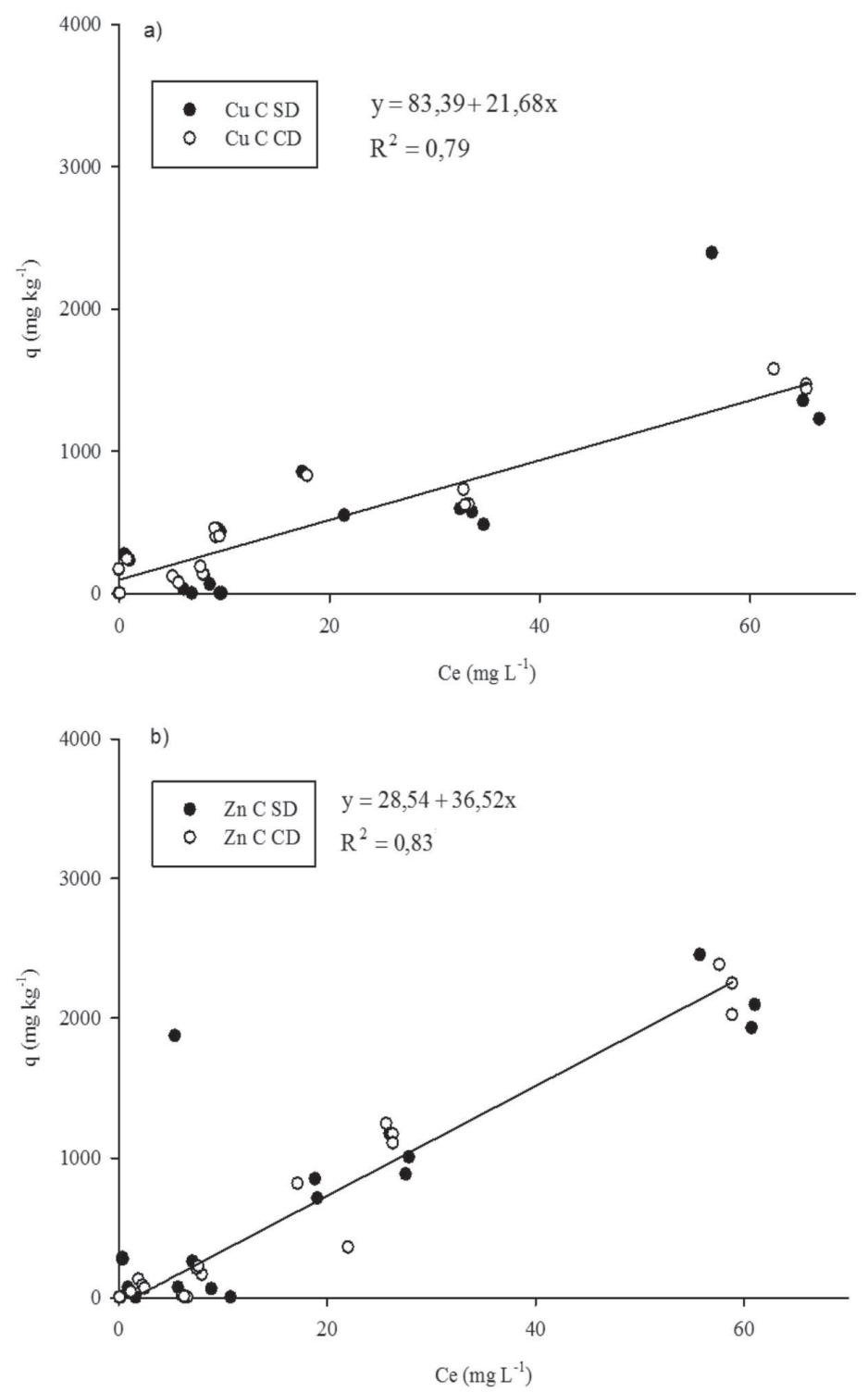

Figura 2. Isotermas de adsorção de $\mathrm{Cu}$ e $\mathrm{Zn}$ competindo a pH 5,5 e força iônica $15 \mathrm{mmol} \mathrm{L}^{-1}$ em Latossolo. a) Adsorção de $\mathrm{Cu}$ competindo no tratamento sem dejeto suíno (SD) e com dejeto (CD); b) Adsorção de Zn competindo no tratamento sem dejeto suíno (SD) e com dejeto (CD). 
na Ce de $60 \mathrm{mg} \mathrm{L}^{-1}$ do tratamento CD (Figura 1b), a adsorção já se aproximava de $1.700 \mathrm{mg} \mathrm{kg}^{-1}$, antes de alcançar o patamar de estabilização. A alta capacidade dos Latossolos de adsorverem $\mathrm{Cu}$ e $\mathrm{Zn}$ e a baixa mobilidade desses elementos pôde ser verificada, por diversos autores. Bertol et al. (2010), avaliando o efeito da aplicação de adubação mineral e de dejetos suínos, em Latossolo Vermelho eutroférrico, observaram baixa lixiviação de $\mathrm{Cu}$ e $\mathrm{Zn}$, independentemente do adubo utilizado. Da mesma forma, Anjos \& Mattiazzo (2000) não observaram lixiviação de $\mathrm{Cu}$ e $\mathrm{Zn}$, em Latossolo Vermelho distrófico e Latossolo Amarelo distrófico tratados com biossólido e atribuíram isso ao poder de acumulação desses metais em Latossolos. Scherer et al. (2010) encontraram menor mobilidade de $\mathrm{Cu}$ e $\mathrm{Zn}$ em Latossolo que em Neossolo e Cambissolo, avaliando o efeito do uso prolongado de dejetos suínos em áreas agrícolas.

Entretanto, apesar da baixa mobilidade do $\mathrm{Cu}$ e $\mathrm{Zn}$ no perfil de Latossolos, deve ser considerado que o acúmulo desses metais na superfície do solo pode ocasionar a contaminação dos mananciais de água, por meio do escoamento superficial desses elementos com a erosão do solo.

\section{CONCLUSÕES}

A aplicação de dejetos suínos resultou em mudança do comportamento de adsorção do $\mathrm{Cu}$, ou seja, no solo que não recebeu aplicação desses dejetos houve o ajuste da isotermo tipo $\mathrm{S}$ aos dados de adsorção de $\mathrm{Cu}$, enquanto, no solo que recebeu dejetos, a isoterma ajustada foi do tipo C;

A capacidade máxima de adsorção de $\mathrm{Cu}$ para solos sem dejetos foi de $3021 \mathrm{mg} \mathrm{kg}^{-1}$.

Para Zn, todas as isotermas apresentaram comportamento linear.

No sistema competitivo, o Cu obteve preferência pelas cargas do solo.

\section{REFERÊNCIAS}

Agbenin JO \& Olojo LA (2004) Competitive adsorption of copper and zinc by a Bt horizon of savanna Alfisol as affected by $\mathrm{pH}$ and selective removal of hydrous oxides and organic matter. Geoderma, 119:85-95.

Almeida JA, Torrent J \& Barrón V (2003) Cor de solo, formas de fósforo e adsorção de fosfatos em Latossolos desenvolvidos de basalto no extremo-sul do Brasil. Revista Brasileira de Ciência do Solo, 27:985-1002.

Anjos ARM \& Mattiazzo ME (2000) Lixiviação de íons inorgânicos em solos repetidamente tratados com biossólido. Revista Brasileira de Ciência do Solo, 24:927-938.

Atsdr - Agency for toxic substances and disease registry (2004) Toxicological profile of copper. Georgia: U.S. Department of health and human services. Disponível em: < www.atsdr.cdc.gov >. Acessado em: 20 de janeiro de 2009.
Atsdr - Agency for toxic substances and disease registry (2005) Toxicological profile of zinc. Georgia: U.S. Department of health and human services. Disponível em: < www.atsdr.cdc.gov >. Acessado em: 20 de janeiro de 2009.

Bertol OJ, Fey E, Favaretto N, Lavoranti J \& Rizzi NE (2010) Mobilidade de $\mathrm{P}, \mathrm{Cu}$ e $\mathrm{Zn}$ em colunas de solo sob sistema de semeadura direta submetido às adubações mineral e orgânica. Revista Brasileira de Ciência do Solo, 34:1841-1850.

Bradl HB (2004) Adsorption of heavy metal ions on soils and soils constituents. Journal of Colloid and Interface Science, 277:01-18.

Casagrande JC, Soares MR \& Mouta ER (2008) Zinc adsorption in highly weathered soils. Pesquisa Agropecuária Brasileira, 43:131-139.

Costa AC (2011) Rendimento de milho, atributos químicos e formas do fósforo no solo após nove aplicações anuais de dejeto suíno. Dissertação de Mestrado. Universidade do Estado de Santa Catarina, Lages. 85p.

Cunha KPV, Nascimento CWA, Pimentel RMM, Accioly AMA \& Silva AJ (2008) Disponibilidade, acúmulo e toxidez de cádmio e zinco em milho cultivado em solo contaminado. Revista Brasileira de Ciência do Solo, 32:1319-1328.

Dudka S, Piotrowska M \& Chlopecka A (1994) Effect of elevated concentrations of $\mathrm{Cd}$ and $\mathrm{Zn}$ in soil on spring wheat yield and the metal content of the plants. Water Air Soil Pollution,76:333-341.

Embrapa - Empresa Brasileira de Pesquisa Agropecuária (1997) Serviço Nacional de Levantamento e Classificação dos Solos. Manual de métodos de análise de solo. $2^{\mathrm{a} e d . ~ R i o ~ d e ~ J a n e i r o, ~ E m b r a p a . ~ 212 p . ~}$

FATMA - Fundação do Meio Ambiente de Santa Catarina (2004) Instrução normativa $\mathrm{n}^{\circ} 11$, de 13/12/2004. Disponível em: <www.fatma.sc.gov.br>. Acessado em: 10 de agosto de 2008.

Giles CH, Smith D \& Huitson A (1974) A general treatment and classification of the solute adsorption isotherm. I: Theoretical. Journal of Colloid and Interface Science, 47:755-765.

Girotto E (2007) Cobre e zinco no solo sob uso intensivo de dejeto líquido de suínos. Dissertação de Mestrado. Universidade Federal de Santa Maria, Santa Maria. 121p.

Girotto E, Ceretta CA, Brunetto G, Santos DR, Silva LS, Lorenzi F, Lorensini F, Viera RCB \& Schmatz R (2010) Acúmulo e formas de cobre e zinco no solo após aplicações sucessivas de dejeto líquido de suínos. Revista Brasileira de Ciência do Solo, 33:955-965.

Gupta CU (2001) Micronutrientes e Elementos Tóxicos em Plantas e Animais. In: Ferreira ME, Cruz MCP, Raij BV \& Abreu CA. Micronutrientes e Elementos Tóxicos na Agricultura. Jaboticabal, CNPQ/FAPESP/POTAFOS. 191p.

Han FX, Kingery WL, Selim HM \& Gerard PD (2000) Accumulation the heavy metals in a long-term poultry waste amended soil. Soil Science, 165:260-268.

Hinz C (2001) Description of sorption data with isotherm equations. Geoderma, 99:225-243.

L'Herrox L, Roux SL, Apprio UP \& Martinez J (1997) Behavior of metals following intensive pig slurry applications to a natural field treatment process in Brittany (France). Environmental Pollution, 97:119-130.

Limousin G, Gaudet JP, Charlet L, Szenknect S, Barthès V \& Krimissa (2007) Sorption isotherms: A review of physical bases, modeling and measurement. Applied Geochemistry, 22:249-275.

López-Alonso M, Benedito JL, Miranda M, Castillo C, Hernández J \& Shore RF (2000) The effect of pig farming on copper and zinc accumulation in Catlle in Galicia (North-Western Spain). The Veterinay Journal, 160:259-266.

Mcbride MB (1994) Environmental chemistry of soil. 1 aed. New York, Oxford University Press. 341p. 
Moreira CS (2004) Adsorção competitiva de cádmio, cobre, níquel e zinco em solos. Dissertação de Mestrado. Escola Superior de Agricultura "Luiz de Queiroz”, Piracicaba. 108p.

Moreira CS \& Alleoni LRF (2010) Adsorption of Cd, Cu, Ni and Zn in tropical soils under competitive and non-competitive systems. Scientia Agricola, 67:301-307.

Nascimento CWA \& Fontes RLF (2004) Correlação entre características de latossolos e parâmetros de equações de adsorção de cobre e zinco. Revista Brasileira de Ciência do Solo, 28:965-971.

NCR - National Reserch Council (1998) Nutrient Requirements of Swine. $10^{\mathrm{a} e d . ~ W a s h i n g t o n, ~ N a t i o n a l ~ A c a d e m y ~ P r e s s . ~ 189 p . ~}$

Paulín RVR, Alberich EMV, Fuente JL \& Mancera ZHA (2006) Eûect of sewage Sludge or compost on the sorption and distribution of copper and cadmium in soil. Waste Management, 26:71-81.

Pierangeli MAP, Guilherme LRG, Curi N, Costa ETS, Lima JM, Marques JJGSM \& Figueiredo LFP (2007) Comportamento sortivo, individual e competitivo, de metais pesados em Latossolos com mineralogia contrastantes. Revista Brasileira de Ciência do Solo, 31:819-826.

SAS Institute Inc® (2003) Statistical Analysis System. Versão 9.1.3. Cary, SAS Institute Inc. CD ROM.

Saviozzi A, Levi-Minzi R, Riffaldi R \& Vanni G (1997) Laboratory studies on application of wheat straw and pig slurry to soil and the resulting environmental implications. Agriculture, Ecosystems \& Environment, 61:35-43
Seganfredo MA (2007) Uso de dejetos suínos como fertilizantes e seus riscos ambientais. In: Seganfredo MA. Gestão Ambiental na Suinocultura. Brasília, Embrapa Informação Tecnológica. p.149-176.

Scherer EE, Nesi CN \& Massotti Z (2010) Atributos químicos do solo influenciados por sucessivas aplicações de dejetos suínos em áreas agrícolas de Santa Catarina. Revista Brasileira de Ciência do Solo, 34:1375-1383.

Schmidt V, Gottardi CPT \& Nadvorny A (2007) Segurança sanitária durante a produção, o manejo e produção, o manejo e a disposição final de dejetos suínos. In: Seganfredo MA (Ed.) Gestão Ambiental na Suinocultura. Brasília, Embrapa Informação Tecnológica. p.251-258.

Silva DCPR (2009) Rendimento de milho e atributos químicos em Latossolo Vermelho fertilizado com adubo solúvel e dejeto suíno. Dissertação de Mestrado. Universidade Estadual de Santa Catarina, Lages. 71p.

Silveira ML, Alleoni LRF \& Chang A (2008) Condicionadores químicos de solo e retenção e distribuição de cádmio, zinco e cobre em Latossolos tratados com biossólido. Revista Brasileira de Ciência do Solo, 32:1087-1088.

Sparks DL (1995) Environmental soilchemistry. San Diego, Academic Press. 267p.

Sposito G (2008)The chemistry of soils. $2^{\mathrm{a}} \mathrm{ed}$. New York, Oxford University Presss. 329p.

Tedesco MJ, Gianello C \& Bissani CA (1995) Análise de solo, plantas e outros materiais. Porto Alegre, Universidade Federal do Rio Grande do Sul. 174p. 